\title{
WET ZINC OXIDE NANOPOWDER CATALYZED EFFICIENT CONVERSION OF ALDEHYDES INTO NITRILES UNDER SOLVENT-FREE CONDITIONS
}

\author{
P. E. More ${ }^{\varpi}$ and S. L. Khillare \\ Department of Chemistry, Agricultural Development Trust's, Sharadabai Pawar Mahila Arts, \\ Comm. \& Sci. College, Shardanagar, Baramati,413115, District Pune, Maharashtra, India. \\ ${ }^{\square}$ Corresponding Author: drpemore@gmail.com
}

\begin{abstract}
The solvent-free approach has been developed for the direct conversion of aldehydes into cyanides using wet zinc oxide nanopowder as a catalyst. The wetness of heterogeneous catalysts is established for the conversion. Compared with other catalytic methods, the current procedure has certain advantages such as short reaction time, high yield of products, solvent-free reaction conditions, non-toxic and commercially available catalyst. The catalyst was recovered by simple filtration and reused in three successive reactions.

Keywords: Solvent-free, Zinc Oxide, Nanopowder, Moisture, Aldehydes, Nitriles.
\end{abstract}

RASĀYAN J. Chem., Vol. 14, No.3, 2021

\section{INTRODUCTION}

Nitriles are one of the important classes of organic compounds. They displayed a key role in the manufacture of dyes, pharmaceuticals, agrochemicals, bioactive natural products and functional materials. ${ }^{1-2}$ In addition; these compounds are used for the preparation of amines, amides, carboxylic acids and nitrogen-containing heterocycles. ${ }^{3}$ Besides, the cyano group itself is present in the biologically active compound, such as Citalopram (anti-depression drug), Letrazole (anti-estrogen drug), Periciazine ( anti-psychotic drug) (Fig.-1), 5-lipoxygenase inhibitors and HIV protease inhibitors. ${ }^{4-6}$
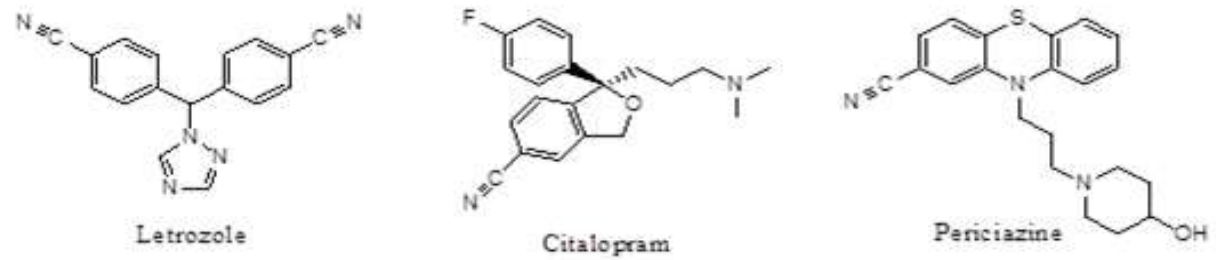

Fig.-1: Some marketed Drugs containing Nitrile Functionality

A huge number of methods have been reported for the preparation of nitriles using a variety of reagents. The classical approaches involve Rosenmund-von Braun and Sandmeyer reactions, but these methods required functionalized starting material and stoichiometric quantity of copper (I) cyanide reagent. ${ }^{711}$ These compounds are prepared on an industrial scale by ammoxidation of toluene derivatives. ${ }^{12}$ However, this method is suitable only for a very short number of substituted toluenes, demands high reaction temperature and pressure, and the use of the large quantity of ammonia restricted its application. Recently, improved synthesis of nitriles starting from aldehydes has been reported using a variety of reagents and catalysts such as $\mathrm{PhI}(\mathrm{OAc})_{2} / \mathrm{SDS}^{13}, \mathrm{KF} / \mathrm{Al}_{2} \mathrm{O}_{3} / \mathrm{DMSO}^{14}, \mathrm{KI} / \mathrm{I}_{2}-\mathrm{TBHP}^{15}, \mathrm{NaN}_{3} / \mathrm{TfOH}^{16}$, $\mathrm{TEMPO} / \mathrm{HNO}_{3} / \mathrm{AcOH}^{17}, \quad \mathrm{HMD}^{18}, \quad \mathrm{CF}_{3}-\mathrm{BHA} / \mathrm{TFA}^{19}, \quad \mathrm{NH}_{4} \mathrm{OH} . \mathrm{HCl} / \mathrm{DMSO}^{20}, \quad \mathrm{EtOPOCl}{ }_{2} / \mathrm{DBU}^{21}$,

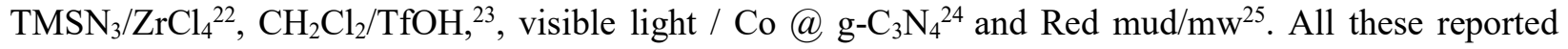
methods have one or other disadvantages, such as the use of expensive, toxic or corrosive reagents, use of volatile organic solvent, drastic reaction conditions, limited scope of the substrate, tedious workup procedure and unsatisfactory yields. Zinc oxide is an inexpensive, environmentally benign and abundantly available inorganic solid. ${ }^{26}$ Commercially available zinc oxide nanopowder is non-toxic and found 
RASĀYAN J. Chem.

Vol. 14 | No. 3 |1829-1834| July - September | 2021

suitable for photocatalytic activity; it could be useful for the manufacture of future generation optoelectronic devices in the U.V region. ${ }^{27-28}$ In the last decade, zinc oxide nano-particles have shown tremendous applications as a reusable heterogeneous catalyst for the various organic transformations. ${ }^{29}$

\section{EXPERIMENTAL}

The $\mathrm{ZnO}$ nanopowder (particle size $<100 \mathrm{~nm}$, surface area $10-25 \mathrm{~m}^{2} / \mathrm{g}$ ) was purchased from Merck and used with no further purification. All reagents were purchased and used without refinement from Fluka. The physical constants (m.p/b.p) were taken in an open capillary tube and are not corrected. Thin-layer chromatography was performed on Merck silica gel plates and spot visualized by U.V. light or exposure to iodine vapors. NMR spectra were obtained using Varian Mercury $\mathrm{YH}-500 \mathrm{MHz}$ spectrometer $\left({ }^{1} \mathrm{H}\right.$ $\mathrm{NMR}$ at $500 \mathrm{MHz}$ and ${ }^{13} \mathrm{C} \mathrm{NMR}$ at $100 \mathrm{MHz}$ ) with TMS as an internal standard; coupling constants are measured in Hz. Infrared spectra were recorded on Shimadzu FT-IR Tracer-100 spectrometer scanning 32 times from $4000-400 \mathrm{~cm}^{-1}$ at $2.5 \mathrm{~cm}^{-1}$ resolution.

\section{General Procedure (2a - 2p)}

Aldehyde (2 mmol), hydroxylamine hydrochloride $(2.5 \mathrm{mmol})$, ZnO nanopowder $0.32 \mathrm{~g}(20 \mathrm{~mol} \%)$ and water ( 2 drops) was taken in a $50 \mathrm{~mL}$ round bottom flask and stirred for $5 \mathrm{~min}$. at room temperature. Then, the temperature was raised to $90^{\circ} \mathrm{C}$ and maintained for the appropriate time (Table 2). The development of the reaction was monitored by TLC. After completion, the reaction mixture was extracted with dichloromethane $(3 \times 5 \mathrm{~mL})$, and the extract was washed with aq. $\mathrm{NaHCO}_{3}$. The organic layer was dried over anhydrous $\mathrm{Na}_{2} \mathrm{SO}_{4}$ and removal of the solvent under reduced pressure furnished analytically pure product.

\section{Spectroscopic Data of Synthesized Compounds \\ Benzonitirle (2a)}

Colorless liquid; bp 190-192 $\mathrm{C}\left(\right.$ lit. $\left.{ }^{25} 187-190^{\circ} \mathrm{C}\right)$; IR (Neat) $2229 \mathrm{~cm}^{-1} ;{ }^{1} \mathrm{H}$ NMR $\left(\mathrm{CDCl}_{3}, 500 \mathrm{MHz}\right): \delta=$ 7.40-7.49m(2H, ArH), 7.55-7.71m(3H, $\mathrm{ArH}) ;{ }^{13} \mathrm{C} \mathrm{NMR}\left(\mathrm{CDCl}_{3}, 100 \mathrm{MHz}\right): \delta=134,131,127,118,112$.

\section{4-Chlorobenzonitrile(2b)}

White solid; mp 90-91 ${ }^{0} \mathrm{C}\left(\right.$ lit. $\left.^{25} 91-193^{0} \mathrm{C}\right)$; IR (Neat) $2230 \mathrm{~cm}^{-1} ;{ }^{1} \mathrm{H}$ NMR $\left(\mathrm{CDCl}_{3}, 500 \mathrm{MHz}\right): \delta=7.54-$ $7.66 \mathrm{~d}(\mathrm{~J} 8.6 \mathrm{~Hz}, 2 \mathrm{H}, \mathrm{ArH}), 7.70-7.81 \mathrm{~d}(\mathrm{~J} 8.9 \mathrm{~Hz}, 2 \mathrm{H}, \mathrm{ArH}) ;{ }^{13} \mathrm{C} \mathrm{NMR}\left(\mathrm{CDCl}_{3}, 100 \mathrm{MHz}\right): \delta=139.1,132.3$, $128.7,118.5,111.3$.

\section{4-Nitrobenzonitrile(2c)}

White solid; mp $149^{0} \mathrm{C}$ (lit. $\left.{ }^{25} 146-149^{0} \mathrm{C}\right)$; IR (Neat) $2231 \mathrm{~cm}^{-1} ;{ }^{1} \mathrm{H}$ NMR $\left(\mathrm{CDCl}_{3}, 500 \mathrm{MHz}\right): \delta=7.89-$ $8.01(\mathrm{~d}, \mathrm{~J}=8.9 \mathrm{~Hz}, 2 \mathrm{H}, \mathrm{ArH}), 8.26-8.40(\mathrm{~d}, \mathrm{~J}=8.9 \mathrm{~Hz}, 2 \mathrm{H}, \mathrm{ArH}) ;{ }^{13} \mathrm{C} \mathrm{NMR}\left(\mathrm{CDCl}_{3}, 100 \mathrm{MHz}\right): \delta=151.0$, $132.9,124.5,118.3,116.9$

\section{3-Nitrobenzonitrile(2d)}

Yellow solid; mp1 $14^{0} \mathrm{C}\left(\right.$ lit. $\left.{ }^{25} 115-117^{0} \mathrm{C}\right)$; IR (Neat) $2234 \mathrm{~cm}^{-1} ;{ }^{1} \mathrm{H}$ NMR $\left(\mathrm{CDCl}_{3}, 500 \mathrm{MHz}\right): \delta=7.57-$

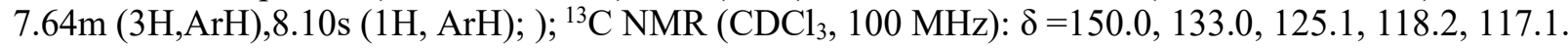

\section{3-Chlorobenzonitrile(2e)}

Pale yellow solid; mp 44-46 ${ }^{0} \mathrm{C}\left(\mathrm{lit}^{25} 43-46{ }^{0} \mathrm{C}\right)$; IR (Neat) $2225 \mathrm{~cm}^{-1} ;{ }^{1} \mathrm{H}$ NMR $\left(\mathrm{CDCl}_{3}, 500 \mathrm{MHz}\right): \delta=$ 7.48-7.53m (2H, ArH),7.73-7.78m (2H, ArH) ); ${ }^{13} \mathrm{C} \mathrm{NMR}\left(\mathrm{CDCl}_{3}, 100 \mathrm{MHz}\right): \delta=139.4,133.3,129.5$, $118.1,110.3$.

\section{4-Brobenzonitrile(2f)}

White solid; mp 114-115 $\mathrm{C}$ (lit. ${ }^{13} 115-117^{0} \mathrm{C}$ ); IR (Neat) $2227 \mathrm{~cm}^{-1} ;{ }^{1} \mathrm{H}$ NMR $\left(\mathrm{CDCl}_{3}, 500 \mathrm{MHz}\right): \delta=$ 7.79-7.91d (J 8.8 Hz, 2H, ArH),8.01-8.25d (J $8.8 \mathrm{~Hz}, 2 \mathrm{H}, \mathrm{ArH}) ;{ }^{13} \mathrm{C} \mathrm{NMR}\left(\mathrm{CDCl}_{3}, 100 \mathrm{MHz}\right): \delta=134.2$, $132.7,128.1,118.2,112$.

\section{2-Hhydroxybenzonitrile(2g)}

Colorless solid; mp 90-92 ${ }^{\circ} \mathrm{C}$ (lit. ${ }^{31} 96{ }^{0} \mathrm{C}$ ); IR (Neat) $2234 \mathrm{~cm}^{-1} ;{ }^{1} \mathrm{H}$ NMR $\left(\mathrm{CDCl}_{3}, 500 \mathrm{MHz}\right): \delta=6.95-$ 7.03m (2H, ArH),7.63-7.79m (2H, $\mathrm{ArH}) ;{ }^{13} \mathrm{C} \mathrm{NMR}\left(\mathrm{CDCl}_{3}, 100 \mathrm{MHz}\right): \delta=160.1,134.2,119.1,116.3$, 103.1 . 
RASĀYAN J. Chem.

Vol. 14 | No. 3 |1829-1834| July - September | 2021

\section{2-Hhydroxy-3-methoxy-benzonitrile (2h)}

Solid; mp57-58 ${ }^{0} \mathrm{C}$ (lit. $\left.{ }^{16} 55-57{ }^{0} \mathrm{C}\right)$; IR (Neat) $2234 \mathrm{~cm}^{-1} ;{ }^{1} \mathrm{H}$ NMR $\left(\mathrm{CDCl}_{3}, 500 \mathrm{MHz}\right): \delta=4.02 \mathrm{~s}(3 \mathrm{H}, \mathrm{Ar}-$ $\left.\mathrm{OCH}_{3}\right), 7.30-7.36 \mathrm{~m}(2 \mathrm{H}, \mathrm{ArH}), 8.79 \mathrm{~s}(1 \mathrm{H}, \mathrm{ArH}) ;{ }^{13} \mathrm{C} \mathrm{NMR}\left(\mathrm{CDCl}_{3}, 100 \mathrm{MHz}\right): \delta=153.3,146.7,145.0$, $125.1,123.5,117.3,112.1,57.3$.

\section{4-Methylbenzotitile(2i)}

White solid; mp34-35 $5^{\circ} \mathrm{C}$ (lit. $\left.{ }^{16} 27-29^{0} \mathrm{C}\right)$; IR (Neat) $2226 \mathrm{~cm}^{-1}$; ${ }^{1} \mathrm{H} \mathrm{NMR}\left(\mathrm{CDCl}_{3}, 500 \mathrm{MHz}\right): \delta=2.79 \mathrm{~s}(3 \mathrm{H}$, Ar- $\left.\mathrm{CH}_{3}\right), 7.7 .30-7.34 \mathrm{~d}(\mathrm{~J} 8.4 \mathrm{~Hz}, 2 \mathrm{H}, \mathrm{ArH}), 7.54-7.58 \mathrm{~d}(\mathrm{~J} 8.4 \mathrm{~Hz}, 2 \mathrm{H}, \mathrm{ArH}) ;{ }^{13} \mathrm{C} \mathrm{NMR}\left(\mathrm{CDCl}_{3}, 100\right.$ $\mathrm{MHz}): \delta=144,132,119.2,109.2,21.9$.

\section{4-Methoxybenzotitile(2j)}

Colorless solid; mp 56-57 ${ }^{0} \mathrm{C}$ (lit. ${ }^{25} 57-59^{0} \mathrm{C}$ ); IR (Neat) $2225 \mathrm{~cm}^{-1} ;{ }^{1} \mathrm{H} \mathrm{NMR}\left(\mathrm{CDCl}_{3}, 500 \mathrm{MHz}\right): \delta=3.94 \mathrm{~s}$ $\left(3 \mathrm{H}, \mathrm{Ar}-\mathrm{OCH}_{3}\right), 7.01 \mathrm{~d}(\mathrm{~J} 8.2 \mathrm{~Hz}, 2 \mathrm{H}, \mathrm{ArH}), 7.67 \mathrm{~d}(\mathrm{~J} 8.2 \mathrm{~Hz}, 2 \mathrm{H}, \mathrm{ArH}) ;{ }^{13} \mathrm{C} \mathrm{NMR}\left(\mathrm{CDCl}_{3}, 100 \mathrm{MHz}\right): \delta$ $=162.7,132,119.1,115,133.7,54.9$.

\section{Cinnamonitrile(2k)}

Colorless oil; bp254-257 ${ }^{0} \mathrm{C}$ (lit. ${ }^{13} 251-252^{0} \mathrm{C}$ ); IR (Neat) $2204 \mathrm{~cm}^{-1} ;{ }^{1} \mathrm{H}$ NMR $\left(\mathrm{CDCl}_{3}, 500 \mathrm{MHz}\right): \delta$ $=6.98 \mathrm{~d}(\mathrm{~J} 9 \mathrm{~Hz}, 2 \mathrm{H}, \mathrm{ArH}), 7.28-7.36 \mathrm{~m}(3 \mathrm{H}, \mathrm{ArH}), 7.49 \mathrm{~d}(\mathrm{~J} 15.9 \mathrm{~Hz}, 1 \mathrm{H},=\mathrm{CH}), 7.78 \mathrm{~d}(\mathrm{~J} 15.9 \mathrm{~Hz}, 1 \mathrm{H},=\mathrm{CH})$;

${ }^{13} \mathrm{C} \mathrm{NMR}\left(\mathrm{CDCl}_{3}, 100 \mathrm{MHz}\right): \delta=150.7,133.2,131.5,129.2,127.3,118.5,96.2$.

\section{Thiophene-2-carbonitrile(2l)}

Colorless liquid; bp $188-190^{\circ} \mathrm{C}$ (lit. ${ }^{25} 134^{\circ} \mathrm{C}$ ); IR (Neat) $2206 \mathrm{~cm}^{-1} ;{ }^{1} \mathrm{H}$ NMR $\left(\mathrm{CDCl}_{3}, 500 \mathrm{MHz}\right): \delta=7.20$ 7.25m (1H,ArH), 7.67-7.75m (2H,ArH); ${ }^{13} \mathrm{C} \mathrm{NMR}\left(\mathrm{CDCl}_{3}, 100 \mathrm{MHz}\right): \delta=138.2,133.1,127.5,117.0$, $114.5,109.1$.

\section{3-Pyridinecarbonitrile(2m)}

Faint yellow solid; mp $50^{\circ} \mathrm{C}\left(\right.$ lit. $\left.{ }^{25} 50^{\circ} \mathrm{C}\right)$; IR (Neat) $2230 \mathrm{~cm}^{-1} ;{ }^{1} \mathrm{H}$ NMR $\left(\mathrm{CDCl}_{3}, 500 \mathrm{MHz}\right): \delta=8.82 \mathrm{~m}($ $3 \mathrm{H}, \mathrm{ArH}), 8.96 \mathrm{~m}(1 \mathrm{H}, \mathrm{ArH}) ;{ }^{13} \mathrm{C} \mathrm{NMR}\left(\mathrm{CDCl}_{3}, 100 \mathrm{MHz}\right): \delta=152.9,152.7,138.9,124.0,117.1,110.2$.

\section{1-Naphthonitrile(2n)}

Faint yellow solid; mp 33-35 $5^{0} \mathrm{C}$ (lit. ${ }^{25} 115-117^{0} \mathrm{C}$ ); IR (Neat) $2224 \mathrm{~cm}^{-1} ;{ }^{1} \mathrm{H}$ NMR $\left(\mathrm{CDCl}_{3}, 500 \mathrm{MHz}\right): \delta$ $=7.73-7.81 \mathrm{~m}(4 \mathrm{H}, \mathrm{ArH}), 7.94-8.02 \mathrm{~m}(3 \mathrm{H}, \mathrm{ArH}) ;{ }^{13} \mathrm{C} \mathrm{NMR}\left(\mathrm{CDCl}_{3}, 100 \mathrm{MHz}\right): \delta=133.5,132.7,132.2$, $132.0,128.4,127.7,125.0,124.7,117.9,110.4$.

\section{Butanenitrile(2o)}

Colorless liquid; bp $118-119^{0} \mathrm{C}\left(\right.$ lit. $\left.^{31} 118^{0} \mathrm{C}\right)$; IR (Neat) $2246 \mathrm{~cm}^{-1} ;{ }^{1} \mathrm{H}$ NMR $\left(\mathrm{CDCl}_{3}, 500 \mathrm{MHz}\right): \delta=0.96 \mathrm{t}$ $\left(\mathrm{J} 2.8 \mathrm{~Hz}, 3 \mathrm{H}, \mathrm{CH}_{3}\right), 1.75 \mathrm{~m}\left(2 \mathrm{H}, \mathrm{CH}_{2}\right), 2.35 \mathrm{t}\left(\mathrm{J} 2.8 \mathrm{~Hz}, 2 \mathrm{H}, \mathrm{CH}_{2}\right) ;{ }^{13} \mathrm{C} \mathrm{NMR}\left(\mathrm{CDCl}_{3}, 100 \mathrm{MHz}\right): \delta=$ $118.9,26.0,25.1,21.3$.

\section{2-Methylpropanenitrile(2p)}

Colorless liquid; bp 106-108 ${ }^{0} \mathrm{C}$ (lit. $\left.{ }^{13} 108-109^{0} \mathrm{C}\right)$; IR (Neat) $2248 \mathrm{~cm}^{-1} ;{ }^{1} \mathrm{H}$ NMR $\left(\mathrm{CDCl}_{3}, 500 \mathrm{MHz}\right): \delta=$ $1.08 \mathrm{~d}\left(\mathrm{~J} 2.9 \mathrm{~Hz}, 6 \mathrm{H}, 2 \mathrm{CH}_{3}\right), 2.43 \mathrm{~m}(1 \mathrm{H}, \mathrm{CH}) ;{ }^{13} \mathrm{C} \mathrm{NMR}\left(\mathrm{CDCl}_{3}, 100 \mathrm{MHz}\right): \delta=119.0,26.3,25.0,21.9$.

\section{RESULTS AND DISCUSSION}

In this protocol, we report the one-pot conversion of aldehydes into nitriles in the presence of catalyst wet zinc oxide nanopowder ( $\mathrm{ZnO}-\mathrm{Np}$ ) under solvent-free conditions (Scheme-1). In the initial experiments, we optimized the reaction conditions using $\mathrm{ZnO}-\mathrm{Np}$ as a catalyst. The reaction between 4-methoxy benzaldehyde $(2 \mathrm{mmol})$ and hydroxylamine hydrochloride $(2.5 \mathrm{mmol})$ was chosen as a model reaction. The results obtained were recorded in Table- 1 . When the reaction mixture was stirred at $70^{\circ} \mathrm{C}$ for 5 hours in an organic reaction medium such as acetonitrile, toluene and dichloromethane furnished a very low yield of the desired product (Table-1 entries 1, 2 and 3). The use of water as solvent at $90^{\circ} \mathrm{C}$ improved the yield but a large amount of aldehyde was recovered unreacted (Table- 1 entries 4 and 5). We previously reported that $\mathrm{ZnO}$ was effective for the synthesis of chalcones and thiol esters under solvent-free conditions. ${ }^{30-31}$ Therefore; we aimed at $\mathrm{ZnO}-\mathrm{Np}$ as a catalyst for this experiment. Then, we attempted to 
RASĀYAN J. Chem.

Vol. 14 | No. 3 |1829-1834| July - September | 2021

carry out the reaction under solvent-free conditions at $90^{\circ} \mathrm{C}$. The result showed that $20 \mathrm{~mol} \%$ of $\mathrm{ZnO}-\mathrm{Np}$, in presence of two drops of water provides a 94\% yield of desired product within a short reaction time (Table-1 entry 8). The additional increase in the amount of catalyst $\mathrm{ZnO}-\mathrm{Np}$ up to $40 \mathrm{~mol} \%$ did not have any significant effect on the product yield or reaction time (Table 1 entry 9 and 10). The incipient moisture could easily release $\mathrm{HCl}$ free from hydroxylamine hydrochloride to dehydrate aldoxime on the surface of a heterogeneous catalyst.
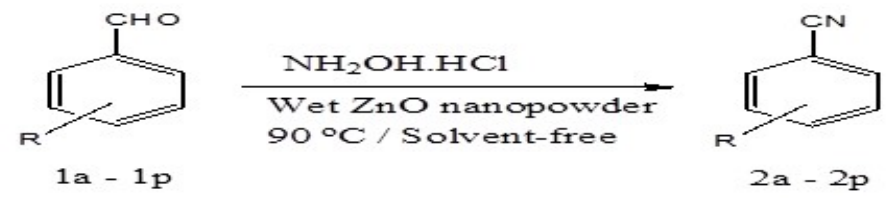

Scheme-1

Table-1: Optimization of Reaction Conditions

\begin{tabular}{c|c|c|c|c|c}
\hline Entry & Catalyst (mol \%) & Solvent & Temperature $\left({ }^{\circ} \mathrm{C}\right)$ & Time (min.) & Yield $^{\mathrm{a}}(\%)$ \\
\hline 1 & 20 & $\mathrm{CH}_{3} \mathrm{CN}$ & 70 & 300 & 20 \\
\hline 2 & 20 & $\mathrm{PhCH}_{3}$ & 70 & 300 & 10 \\
\hline 3 & 20 & $\mathrm{CH}_{2} \mathrm{Cl}_{2}$ & 70 & 300 & 15 \\
\hline 4 & 20 & $\mathrm{H}_{2} \mathrm{O}$ & 90 & 300 & 48 \\
\hline 5 & 40 & $\mathrm{H}_{2} \mathrm{O}$ & 90 & 300 & 47 \\
\hline 6 & 20 & --- & 90 & 45 & 68 \\
\hline 7 & 30 & --- & 90 & 45 & 70 \\
\hline 8 & 20 & --- & 90 & 45 & $94^{\mathrm{b}}$ \\
\hline 9 & 30 & --- & 90 & 45 & $94^{\mathrm{b}}$ \\
\hline 10 & 40 & --- & 90 & 45 & $93^{\mathrm{b}}$ \\
\hline
\end{tabular}

aIsolated yield. ${ }^{b}$ Reactions were carried out using 4-methoxy benzaldehyde ( $\left.2 \mathrm{mmol}\right)$, hydroxylamine hydrochloride $(2.5 \mathrm{mmol})$, catalyst ( $\mathrm{ZnO}-\mathrm{Np})$ and two drops of water.

After determining the best possible reaction conditions, we then examined the scope of reaction for the synthesis of nitriles using various aldehydes. The results are summarized in Table-2. Both aromatic and heteroaromatic aldehydes underwent smooth conversion to give a high yield of products in a short reaction time. However, aliphatic aldehydes provided a lower yield as compared with aromatic aldehydes. All activated (Table-2 entries g, h, i and j) and deactivated (Table- 2 entries b, c, d, e and f) aromatic aldehydes reacted rapidly to furnish corresponding nitriles with excellent yield. The reaction products are clean required no chromatographic separation except in few cases (Table 2 entries o and p). It is noteworthy to mention that our one-pot procedure does not require corrosive $\mathrm{CH}_{3} \mathrm{COCl}$ as observed in dehydration of aldoximes ${ }^{32}$ or reflux in an organic solvent like DMF for 2.30 to 6 hours.

Moreover, the reusability of the catalysis was examined using model reaction for the synthesis of 4methoxy benzonitirle. The catalyst was separated by simple filtration after each reaction, washed with $\mathrm{CH}_{2} \mathrm{Cl}_{2}$, dried in an oven at $100^{\circ} \mathrm{C}$ and tested for activity in the subsequent reaction. It was found that the catalyst could be recycled and reusable three times without loss of activity.

Comparison of the effectiveness of wet $\mathrm{ZnO}-\mathrm{Np}$ catalysts with reported catalysts is shown in Table-3. As compared to reported methods involving the use of hazardous catalyst under volatile reaction medium ${ }^{13-15}$, ${ }^{19}$ the present methodology is superior as excellent yield of products are obtained in short reaction time under solvent-free conditions. Some methods have reported a good yield of products at room temperature. ${ }^{21-24}$ However, the requirement of toxic volatile organic solvent and high reaction time are the weaknesses of these methods.

Table-2: Synthesis of Nitriles $2 a-2 p$ under aqueous under Solvent-free Conditions ${ }^{\mathrm{a}}$

\begin{tabular}{c|l|c|c|c}
\hline Entry & Aldehyde (1) & Product (2) & Time (min.) & Yield (\%) $^{\mathrm{b}}$ \\
\hline $\mathrm{a}$ & $\mathrm{C}_{6} \mathrm{H}_{5}-\mathrm{CHO}$ & $2 \mathrm{a}$ & 48 & 92 \\
\hline $\mathrm{b}$ & $4-(\mathrm{Cl})-\mathrm{C}_{6} \mathrm{H}_{4}-\mathrm{CHO}$ & $2 \mathrm{~b}$ & 40 & 93 \\
\hline $\mathrm{c}$ & $4-\left(\mathrm{NO}_{2}\right)-\mathrm{C}_{6} \mathrm{H}_{4}-\mathrm{CHO}$ & $2 \mathrm{c}$ & 35 & 95 \\
\hline $\mathrm{d}$ & $3-\left(\mathrm{NO}_{2}\right)-\mathrm{C}_{6} \mathrm{H}_{4}-\mathrm{CHO}$ & $2 \mathrm{~d}$ & 50 & 92 \\
\hline $\mathrm{e}$ & $3-(\mathrm{Cl})-\mathrm{C}_{6} \mathrm{H}_{4}-\mathrm{CHO}$ & $2 \mathrm{e}$ & 45 & 90 \\
\hline
\end{tabular}


RASĀYAN J. Chem.

Vol. 14 | No. 3 |1829-1834| July - September | 2021

\begin{tabular}{c|l|l|l|l}
\hline $\mathrm{f}$ & $4-(\mathrm{Br})-\mathrm{C}_{6} \mathrm{H}_{4}-\mathrm{CHO}$ & $2 \mathrm{f}$ & 35 & 94 \\
\hline $\mathrm{g}$ & $2-(\mathrm{OH})-\mathrm{C}_{6} \mathrm{H}_{4}-\mathrm{CHO}$ & $2 \mathrm{~g}$ & 45 & 90 \\
\hline $\mathrm{h}$ & $2-(\mathrm{OH}), 3-\left(\mathrm{OCH}_{3}\right)-\mathrm{C}_{6} \mathrm{H}_{3}-\mathrm{CHO}$ & $2 \mathrm{~h}$ & 48 & 90 \\
\hline $\mathrm{i}$ & $4-\left(\mathrm{CH}_{3}\right)-\mathrm{C}_{6} \mathrm{H}_{4}-\mathrm{CHO}$ & $2 \mathrm{i}$ & 40 & 95 \\
\hline $\mathrm{j}$ & $4-\left(\mathrm{OCH}_{3}\right)-\mathrm{C}_{6} \mathrm{H}_{4}-\mathrm{CHO}$ & $2 \mathrm{j}$ & 45 & 94 \\
\hline $\mathrm{k}$ & $\mathrm{C}_{6} \mathrm{H}_{4}-\mathrm{CH}=\mathrm{CH}-\mathrm{CHO}$ & $2 \mathrm{k}$ & 50 & 91 \\
\hline $\mathrm{l}$ & Thiophene-2-carboxaldehyde & $2 \mathrm{l}$ & 50 & 88 \\
\hline $\mathrm{m}$ & 3-Pyridinecarboxaldehyde & $2 \mathrm{~m}$ & 52 & 91 \\
\hline $\mathrm{n}$ & 1-Naphthaldehyde & $2 \mathrm{n}$ & 50 & 90 \\
\hline $\mathrm{o}$ & Butyraldehyde & $2 \mathrm{o}$ & 80 & 75 \\
\hline $\mathrm{p}$ & Isobutyraldehyde & $2 \mathrm{p}$ & 90 & 70 \\
\hline
\end{tabular}

${ }^{a}$ Reactions were carried out using aldehyde $(2 \mathrm{mmol})$, hydroxylamine hydrochloride $(2.5 \mathrm{mmol}), \mathrm{ZnO}-\mathrm{Np}(20 \mathrm{~mol} \%)$ and two drops of water. ${ }^{b}$ Yield of isolated products.

Table-3: Comparison of wet ZnONanopowder as a Catalyst with reported Catalytic Methods

\begin{tabular}{c|l|c|c|c}
\hline Entry & \multicolumn{1}{|c|}{ Catalyst/Solvent } & $\begin{array}{c}\text { Temp. } \\
\left({ }^{0} \mathrm{C}\right)\end{array}$ & $\begin{array}{c}\text { Time } \\
(\mathrm{h})\end{array}$ & $\begin{array}{c}\text { Yield } \\
(\%)\end{array}$ \\
\hline 1 & $\mathrm{PhI}(\mathrm{OAc})_{2} / \mathrm{SDS}$ & 70 & $1-8$ & $95^{13}$ \\
\hline 2 & $\mathrm{KF} / \mathrm{Al}_{2} \mathrm{O}_{3} / \mathrm{DMSO}$ & 100 & $4.5-10.5$ & $91^{14}$ \\
\hline 3 & $\mathrm{KI} / \mathrm{I}_{2}-\mathrm{TBHP}$ & 60 & 15 & $87^{15}$ \\
\hline 4 & $\mathrm{TEMPO}_{\mathrm{HNO}} / \mathrm{AcOH}$ & 50 & 12 & $91^{17}$ \\
\hline 5 & $\mathrm{CF}_{3}-\mathrm{BHA} / \mathrm{TFA} / \mathrm{DME}$ & 80 & - & $92^{19}$ \\
\hline 6 & $\mathrm{CH}_{2} \mathrm{Cl}_{2} / \mathrm{TfOH}$ & r.t. & 24 & $93^{23}$ \\
\hline 7 & $\mathrm{TMSN}_{3} / \mathrm{ZrCl}_{4} / \mathrm{CH}_{3} \mathrm{CN}$ & r.t. & 24 & $95^{22}$ \\
\hline 8 & $\mathrm{EtOPOCl} / \mathrm{CH}_{2} \mathrm{Cl}_{2}, \mathrm{DBU} / \mathrm{M} . \mathrm{S}$. & r.t. & 15 & $96^{21}$ \\
\hline 9 & $\mathrm{Co}$ a, $\mathrm{g}_{3} \mathrm{~N}_{4} / \mathrm{MeOH}_{2} \mathrm{O}$ & r.t. & $14-20$ & $92^{24}$ \\
\hline 10 & $\mathrm{Wet} \mathrm{ZnO}-\mathrm{Np}_{2} / \mathrm{solvent}-\mathrm{Oree}$ & 90 & $0.35-1.30$ & $95^{\text {This method }}$ \\
\hline
\end{tabular}

All synthesized nitrile products 2a-p were characterized by IR, ${ }^{1} \mathrm{H}$ NMR and ${ }^{13} \mathrm{C}$ NMR spectroscopic methods. The spectral data of almost all synthesized compounds has supported the conversion of aldehydes to nitriles. For instance, compound $2 p$ was characterized by its ${ }^{13} \mathrm{C}$ NMR spectrum which showed characteristics $-\mathrm{CN}$ carbon at $\delta 119.0 \mathrm{ppm}$ which was further confirmed by the IR absorption band at $2248 \mathrm{~cm}^{-1}$. Moreover, the absence of a peak at $\delta 9-10 \mathrm{ppm}$ due to $-\mathrm{CHO}$ proton in the ${ }^{1} \mathrm{H}$ NMR spectra of target compounds $\mathbf{2 a - p}$ indicated the completion of the conversion.

\section{CONCLUSION}

In conclusion, we have developed a simple and competent protocol for the synthesis of nitriles using commercially available, low-cost and environmentally friendly zinc oxide nanopowder as a catalyst under thermal and solvent-free conditions. The advantages of this one-step environmentally benign procedure are recovery and reuse of catalyst, solvent-free reaction conditions and excellent yield of products in a short reaction time.

\section{ACKNOWLEDGEMENT}

The authors are thankful to Chairman, Agricultural Development Trust Baramati, for providing all necessary facilities for this research work.

\section{REFERENCES}

1. F. F. Fleming, L. Yao, P. C. Ravikumar, L. Funk, B. C. Shook, Journal of Medicinal Chemistry, 53(22), 7902 (2010), https://doi.org/10.1021/jm100762r

2. J. S. Miller, J. L. Manson,Accounts of Chemical Research, 34(7), 563 (2001), https://doi.org/10.1021/ar0000354

3. Z. Rappoport, Chemistry of the Cyano Group, London, UK: John Wiley \& Sons, 121 pp 434716(1970), https://doi.org/10.1002/9780470771242.ch11

4. L. H. Jones, N. W. Summerhill, N. A. Swain, J. E. Mills, Medicinal Chemistry Communications,01, 309(2010), https://doi.org/10.1039/C0MD00135J

5. M. N. Janakirman, K. D. Watenpaugh,K. T. Chong, Bioorganic Medicinal Chemistry Letters, 8(10),1237 (1998), https://doi.org/10.1016/S0960-894X(98)00197-8 
RASĀYAN J. Chem.

Vol. 14 | No. 3 |1829-1834| July - September | 2021

6. T. Chatterji, K. S. Gates, Bioorganic Medicinal Chemistry Letters, 8(5), 535 (1998), https://doi.org/10.1016/S0960-894X(98)00066-3

7. T. Sandmeyer, Berichte der deutschenchemischen Gesellschaft, 17,2650 (1884), https://doi.org/10.1002/cber.188401702202

8. C. Galli, Chemical Review, 88, 765(1988), https://doi.org/10.1021/cr00087a004

9. K. W. Rosenmund, E. Struck, Berichte der deutschenn chemischen Gesellschaft (A and B), 52, 17496 (1919), https://doi.org/10.1002/cber.19190520840

10. V. Boosa, V. Bilakanti, N. Gutta, V. K. Velisoju, S. Medak, B. Jorge, K. Muxina, V. Akula, Catalysis Science \& Technology, 6, 8055 (2016), https://doi.org/10.1039/C6CY01536K

11. A. Pradal, G. Evano,Chemical Communications, 50,11907 (2014), https://doi.org/10.1039/C4CC05557H

12. A. Martin, N. V. Kalevaru, B. Lucke,J. Sans, Green Chemistry, 4, 481 (2004), https://doi.org/10.1039/B204244B

13. C. Zhu, L. Ji,Y. Wei,Synthesis, 18, 3121(2010), https://doi.org/10.1055/s-0030-1258162

14. B. Movassagh, S. Shokri, Tetrahedron Letters, 46, 6923(2005), https://doi.org/10.1016/j.tetlet.2005.08.007

15. R. Reddy,C. U. Maheswari, M.Venkateshwar, S. Prashanthi, M. L. Kantam, Tetrahedron Letters, 50,2050 (2009), https://doi.org/10.1016/j.tetlet.2009.02.057

16. B. V. Rokade, K. R. Prabhu,Journal of Organic Chemistry,77, 5364 (2012), https://doi.org/0.1021/jo3008258

17. J. H. Noh, J. Kim, Journal of Organic Chemistry, 80(22),11624 (2015), https://doi.org/10.1021/acs.joc.5b02333

18. C. B. Kelly, K. M. Lambert, M. A. Mercadante, J. M. Ovian, W. F. Bailey, N. E. Leadbeater, Angewandte International Edition Chemie, 54(14), $4241 \quad$ (2015), https://doi.org/10.1002/anie.201412256

19. X.D. An, S. Yu, Organic Letters, 17,5064 (2015), https://doi.org/10.1021/acs.orglett.5b02547

20. J. Augustine, K.A.Bombrun, R. N. Atta, Synlett, 15, 2223 (2011), https://doi.org/10.1055/s-0030$\underline{1261181}$

21. J-L. Zhu, F-Y. Lee, J-D. Wu, C-W. Kuo, K-S. Shia, Synlett, 8,1317 (2007), https://doi.org/10.1055/s2007-980338

22. P. Nimnual, J. Tummatorn, C. Thongsornkleeb, S. Ruchirawat, Journal of Organic Chemistry, 80(17), 8657(2015), https://doi.org/10.1021/acs.joc.5b01305

23. K. Hyodo, K. Togashi, N. Oishi, G. Hasegawa, K. Uchida, Organic Letters, 19(11), 3005(2017), https://doi.org/10.1021/acs.orglett.7b01263

24. F. Vrerma, P. Shukla,S. R. Bhadyia, M. Singh, A. K. Rai, V. K. Rai, Catalysis Communication, 119, 76 (2019), https://doi.org/10.1016/j.catcom.2018.10.031

25. S. H. Khezri, N. Azimi, M. M. Vali, B. E. Sis, M. M. Hashemi, M. H. Baniasadi, F. Teimouric, Arkivoc, xv, 162(2007), https://doi.org/10.3998/ark.5550190.00008.f16

26. K.V. Raju, S. S. Begum, B. D. Sagar, S. Babu, Rasayan Journal of Chemistry, 10(1), 37 (2017), https://doi.org/10.7324/RJC.2017.1011534

27. C. Rani, M. Hemalatha, S. H. Bindu, C. L. Raju, Rasayan Journal of Chemistry, 13(2), 887 (2020), https://doi.org/10.31788/RJC.2020.1325582

28. V. Prasad, G. G. Simiyon, A. E. Mammen, N. Jayaprakash, Rasayan Journal of Chemistry, 12(2), 860 (2019), https://doi.org/10.31788/RJC.2019.1225226

29. B. Banerjee, Journal of Nanostructure in Chemistry, 7, 389 (2017), https://doi.org/10.1007/s40097017-0247-0

30. P. E. More, B. P. Bandgar, V. T. Kamble, Catalysis Communication, 27, 30 (2012), https://doi.org/10.1016/j.catcom.2012.06.012

31. B. P. Bandgar, P. E. More, V. T. Kamble, S. S. Sawant, Australian Journal of Chemistry, 61,1006 (2008), https://doi.org/10.1071/CH08202

32. M. H. Sarvari, Synthesis, 5, 787 (2005), https://doi.org/10.1055/s-2005-861826

[RJC-6410/2021] 